\title{
Polarization-Sensitive and Wide Incidence Angle-Insensitive Fabry-Perot Optical Cavity Bounded by Two Metal Grating Layers
}

\author{
Jehwan Hwang ${ }^{1,2,+}$, Zahyun $\mathrm{Ku}^{3,+}$, Jiyeon Jeon ${ }^{1,4}{ }^{(1)}$, Yeongho Kim ${ }^{1}$, Deok-Kee Kim ${ }^{4}$, \\ Eun Kyu Kim 2,*(D) and Sang Jun Lee ${ }^{1, *}$ \\ 1 Interdisciplinary Materials Measurement Institute, Korea Research Institute of Standards and Science, \\ Daejeon 34113, Korea; wowhwang87@gmail.com (J.H.); jeony210@gmail.com (J.J.); \\ ykim172@kriss.re.kr (Y.K.) \\ 2 Department of Physics and Research Institute for Convergence of Basic Sciences, Hanyang University, \\ Seoul 04763, Korea \\ 3 Materials and Manufacturing Directorate, Air Force Research Laboratory, WPAFB, OH 45433, USA; \\ zahyun.ku.1.ctr@us.af.mil \\ 4 Department of Electronic Engineering, Sejong University, Seoul 05006, Korea; deokkeekim@sejong.ac.kr \\ * Correspondence: ek-kim@hanyang.ac.kr (E.K.K.); sjlee@kriss.re.kr (S.J.L.) \\ + These authors contributed equally to this work.
}

Received: 22 July 2020; Accepted: 18 September 2020; Published: 20 September 2020

\begin{abstract}
Infrared (IR) polarimetric imaging has attracted attention as a promising technology in many fields. Generally, superpixels consisting of linear polarizer elements at different angles plus IR imaging array are used to obtain the polarized target signature by using the detected polarization-sensitive intensities. However, the spatial arrangement of superpixels across the imaging array may lead to an incorrect polarimetric signature of a target, due to the range of angles from which the incident radiation can be collected by the detector. In this article, we demonstrate the effect of the incident angle on the polarization performance of an alternative structure where a dielectric layer is inserted between the nanoimprinted subwavelength grating layers. The well-designed spacer creates the Fabry-Perot cavity resonance, and thereby, the intensity of transverse-magnetic I-polarized light transmitted through two metal grating layers is increased as compared with a single-layer metal grating, whereas transverse-electric (TE)-transmitted light intensity is decreased. TM-transmittance and polarization extinction ratio (PER) of normally incident light of wavelength $4.5 \mu \mathrm{m}$ are obtained with 0.49 and 132 , respectively, as the performance of the stacked subwavelength gratings. The relative change of the PERs for nanoimprint-lithographically fabricated double-layer grating samples that are less than $6 \%$ at an angle of incidence up to $25^{\circ}$, as compared to the normal incidence. Our work can pave the way for practical and efficient polarization-sensitive elements, which are useful for many IR polarimetric imaging applications.
\end{abstract}

Keywords: polarimetric imaging; subwavelength grating; Fabry-Perot cavity; surface plasmon resonance; double-layer metal grating

\section{Introduction}

Infrared (IR) polarimetric imaging systems have been extensively employed in many fields, such as remote sensing, military applications, and environmental protection [1-5]. Polarimetric images provide information based on the polarization signature of radiation instead of detecting optical radiation with scalar fields in traditional infrared images [6,7]. The polarization signature depends on the polarization properties of scattering, reflection, and absorption, which result from the geometry and roughness of 
the target surfaces, and it can be used to distinguish the target from the clutter and background [8-12]. The polarimetric imaging system usually uses a broadband imager for the spatiotemporal information with the mechanical parts of optical components, to obtain the polarimetric signature of a target. However, these mechanical parts make the acquisition of real-time image information difficult, as well as increase the cost and complexity of imagers.

Division-of-focal-plane (DoFP) polarization imaging sensors have been widely studied because of their capability to acquire both the intensity and polarization information in real-time using a monolithically integrated focal plane array (FPA) with a micro-optical element (array of micropolarizers) [13-16]. Typically, the micropolarizer array consists of a $2 \times 2$ array of metallic subwavelength gratings oriented at $0,45,90$, and $135^{\circ}$ to simultaneously determine the first three Stokes parameters [17-20]. Metallic subwavelength gratings are one-dimensional periodic structures with much smaller than the wavelength of the light, which have been recognized as an effective polarizer candidate for the IR regime [21-24]. Specifically, a large portion of linearly polarized incident light perpendicular to the grating direction (TM-, $p$ - or transverse-magnetic polarization) can be transmitted through metallic gratings, while the incident light with polarization parallel to the grating direction (TE-, s- or transverse-electric polarization) is rarely transmitted. The polarization extinction ratio (PER) can be used as the performance indicator of designed polarizers and is defined as the ratio of the transmitted light intensity of perpendicular polarizations (i.e., TM- and TE- polarization).

Recently, a double-layer grating structure has been proposed to improve the polarization detection performance, as well as to reduce the complexity and cost of a lithography technology resulting from simply decreasing the grating period in a single-layer grating structure. As compared to a single-layer grating, the double-layer grating was demonstrated to enhance TM-transmission owing to the multiple reflections, Fabry-Perot (FP) cavity resonance, inside a dielectric spacer layer interposed between two metal gratings, on the other hand, TE-transmitted light intensity through the double-layer grating tends to drastically decrease, leading to a higher PER [25-28].

As mentioned earlier, one of the main advantages of DoFP polarization sensors is the capability of capturing polarization information at every frame by incorporating a pixel-wise polarizer array aligned to an FPA; however, this hybridized configuration can also bring the disadvantages [29-31], such as a loss of spatial resolution (the spatial resolution is decreased by a factor of the number of linear polarizer elements that constitute the superpixel) and inaccuracy of the captured polarization information (each pixel has a varying instantaneous field of view (IFoV), and thereby, it is worth investigating the margin of the validity of a double-layer grating structure used as the linear polarizer element in terms of the angle of incidence within the FoV range). Note that various interpolation methods have been recently proposed to improve both the spatial resolution and the accuracy of the polarization information, due to the sensors' spatially modulated arrangement of a micro-polarization array. These include bilinear, bicubic, bicubic spline, and gradient-based interpolation methods [32-34], new micro-polarizer array patterns-used interpolation method [35,36], spatio-temporal channeled approach [37], smoothness-based interpolation method [38], Newton's polynomials based interpolation [39], correlation-based interpolation method [40], deep convolutional neural network-based polarization demosaicing [41,42], minimized Laplacian polarization residual interpolation [43], sparse representation-based demosaicing method [44], etc.

Here, we focus on experimentally and theoretically analyzing the polarization sensitivity and PER of the double-layered Au grating using the polar and azimuthal angles of incident light on the basis of FoV of the thermal imaging lens. Numerical simulations based on the finite integration technique and the finite element method are carried out to better understand the resonance behavior of the double-layer Au grating, specifically, surface plasmon resonance at the interface between gold and substrate (when stacking the grating layers with a large grating period) and Fabry-Perot resonance. The analysis and modeling results show a good agreement with Fourier transform infrared (FTIR)-measured data of the nanoimprint-lithographically fabricated double-layer Au grating. 


\section{Design of Double-Layer Grating Using a Multiple-Layer Model}

A multiple-layer model [45-47] was employed to efficiently design the double-layer grating structure and to better understand the underlying mechanism of wave propagation inside the spacer layer bounded by two identical gold $(\mathrm{Au})$ grating layers as illustrated in Figure 1a. The overall reflection and transmission coefficients of the double-layer grating can be obtained by a transfer matrix model, which depends on the transmission and reflection of each grating layer. Using the multiplication of the transfer matrix of each layer (i.e., upper and lower grating layers, and spacer layer), the overall transmission coefficient $t$ can be obtained as given by $t=t_{12} t_{23} e^{-i \beta} /\left(1-r_{21} r_{23} e^{-2 i \beta}\right)$ where $r_{21}$ and $r_{23}$ are the reflection coefficients at the backside of upper Au grating and front side of lower Au grating, respectively, and $t_{12}$ and $t_{23}$ are the transmission coefficients through the upper $\mathrm{Au}$ grating and lower Au grating, respectively. $\beta=n_{\text {spacer }} \cdot k \cdot t_{d}$, where $n_{\text {spacer }}$ is the refractive index of spacer, $k$ is the wave vector in free space, $t_{d}$ is the spacer thickness (Figure 1b). The refractive index of the spacer material used for the multiple-layer model was taken as $n_{\text {spacer }}=1.54$, which is close to the measured real part of the refractive index of benzocyclobutane (BCB). Here, to create the Fabry-Perot (FP) cavity resonance inside the spacer of the double-layer grating, the round-trip propagation phase condition $\gamma=\phi_{r_{21}}+\phi_{r_{23}}-2 \beta$ where $\phi_{r_{21}}$ and $\phi_{r_{23}}$ are the phase of $r_{21}$ and $r_{23}$, respectively) is needed to be equal to an integer multiple of $2 \pi$ (Figure 1c). Figure $1 \mathrm{~d}$ shows the TM-transmission colormap, $T\left(\lambda, t_{d}\right)$, obtained by the analytical calculation using the multiple-layer model, and the white dash lines indicate where the FP cavity resonance condition for TM-polarized incidence is satisfied (i.e., $\gamma=-2 \pi,-4 \pi,-6 \pi$ ). In the wavelength of interest (mid-wavelength infrared region, MWIR, 3-5 $\mu \mathrm{m})$, the spectral position of TM-transmission peaks for the double-layer grating $\left(T(0)_{T M}\right.$ in Figure A1) agrees well with the position of FP cavity resonances (Figure 1d). $\gamma=-2 \pi$ (the lowest order of FP cavity resonance) and $t_{d}=0.25 \mu \mathrm{m}$ are the most suitable to realize the broadband linear polarizer covering the MWIR range. Narrowband transmission can be found at the high order FP cavity resonance, as shown in Figure 1d,e, and Figure A1 (an increase in the spacer thickness produces more transmission peaks and makes the transmission bandwidth narrower).

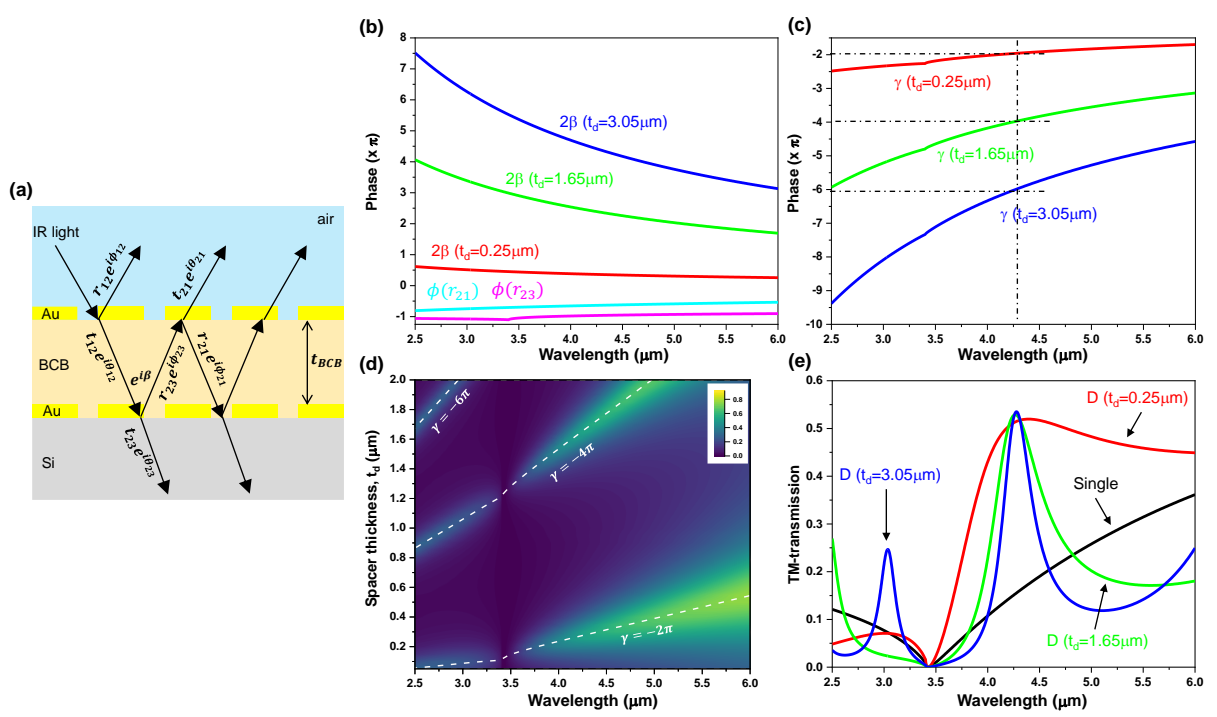

Figure 1. (a) Schematic illustration of the multiple-layer model of the double-layer grating (grating period $p=1.0 \mu \mathrm{m}$; grating width $\left.w=0.7 \mu \mathrm{m} ; t_{\mathrm{Au}}=0.1 \mu \mathrm{m}\right) ;(\mathbf{b})$ phase terms, $\phi\left(r_{21}\right), \phi\left(r_{23}\right), 2 \beta$, used in the Fabry-Perot (FP) cavity resonance condition $\gamma ;(\mathbf{c}) \gamma=\phi\left(r_{21}\right)+\phi\left(r_{23}\right)-2 \beta ;$ (d) transverse-magnetic I-transmission spectra colormap $T\left(\lambda, t_{d}\right)$ obtained by the analytical calculation using the multiple-layer model; (e) reconstructed TM-transmission (based on the multiple-layer model) for the double-layer grating with a spacer thickness of $0.25 \mu \mathrm{m}, 1.65 \mu \mathrm{m}, 3.05 \mu \mathrm{m}$. The FP cavity resonance condition of $\gamma=-2 \pi,-4 \pi,-6 \pi$ is satisfied at $\lambda=\sim 4.3 \mu \mathrm{m}$. 


\section{Theoretical Analysis of Incidence Angle-Dependent Polarization Sensitivity and PER of Double (Single)-Layer Au Grating}

To create test scenarios (for the study of incidence angle-dependent polarization sensitivity and PER of single- and double-layer Au grating structures), we first assumed that the superpixel is composed of a $2 \times 2$ array of single- or double-layer Au gratings oriented at 0 (perpendicular to $\hat{x}$ ), 45, 90 (parallel to $\hat{x}$ ), and $135^{\circ}$ as illustrated in Figure 2. Note that the geometrical parameters (grating period $p$, grating width $w$, Au thickness $t_{\mathrm{Au}}$ ) of the single- and double-layer Au gratings are fixed at $1 \mu \mathrm{m}, 0.7 \mu \mathrm{m}, 0.1 \mu \mathrm{m}$, respectively, and a dielectric spacer $\left(t_{\mathrm{BCB}}=0.25 \mu \mathrm{m}\right)$ was used to separate two Au grating layers in the double-layer structure (Figures 1 and A1-A5). Moreover, we specified two test scenarios to mimic the angles of linearly polarized IR incidence passing through the thermal imaging lens as follows: $k\left(\theta, \phi=0^{\circ}\right)$ and $k\left(\theta, \phi=90^{\circ}\right)$, incident IR rays in a direction described by the spherical coordinates, are characterized by the electric field lying in the plane of incidence ( $x z$-plane) for test scenario A as illustrated in Figure $2 \mathrm{a}, \mathrm{d}$, and the electric field perpendicular to the plane of incidence ( $y z$-plane) for test scenario B as indicated in Figure $2 b, d$, respectively.

(a)

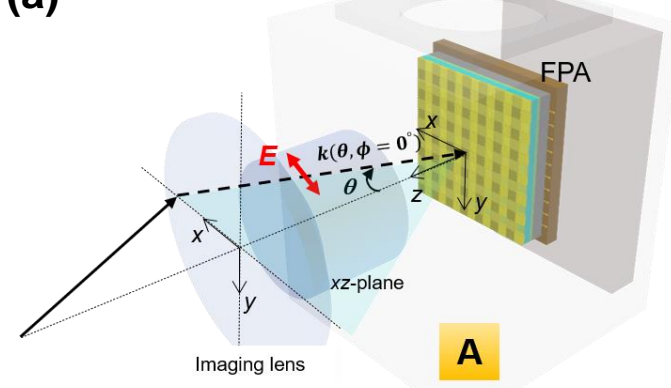

(c)

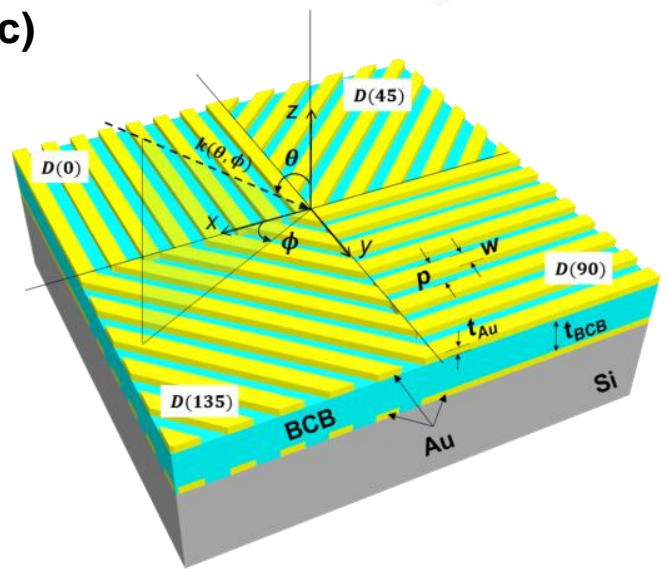

(b)

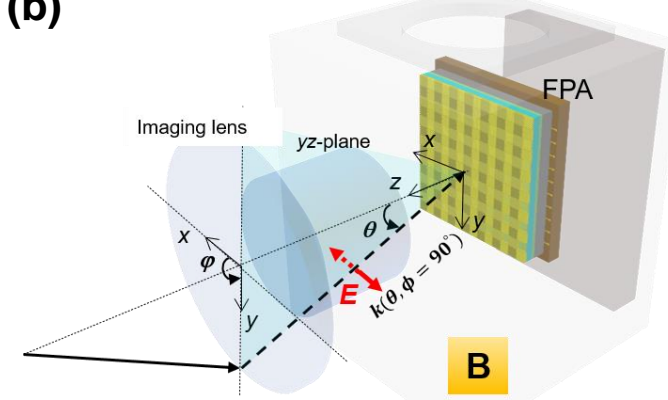

(d)

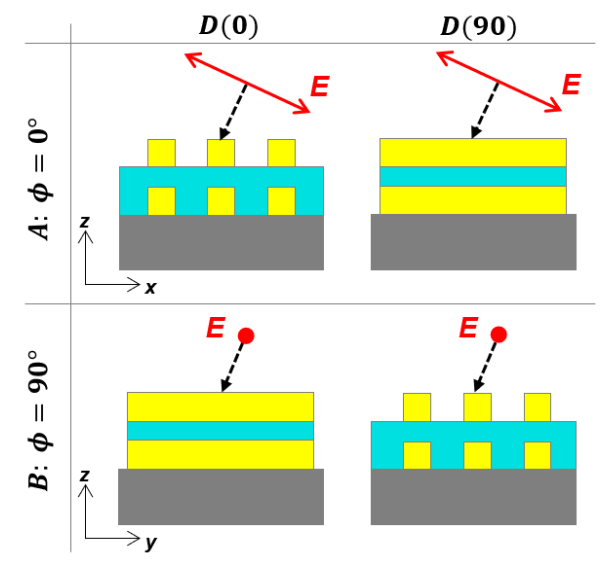

Figure 2. Schematic of the polar $(\theta)$ and azimuth $(\phi)$ angle-dependent infrared (IR) incidence through the thermal imaging lens: Illustrations of (a) test scenario A; (b) test scenario B; (c) three-dimensional configuration of the superpixel consisting of linear polarizer elements at different angles $(0,45,90$, $\left.135^{\circ}\right) . k, \theta$, and $\phi$ denote the incident wave vector, polar angle, and azimuthal angle, respectively; (d) $D(0)$ and $D(90)$ for $\left(\theta, \phi=0\right.$ or $\left.90^{\circ}\right)$.

We performed 3D full field EM simulation (CST Microwave Studio [48]) to theoretically analyze the polarization sensitivity and PER of double-layer $(D)$ and single-layer $(S)$ gold grating structures using the angled incidence. The optical parameters for constitutive materials (i.e., $\mathrm{BCB}$ and silicon ( $\mathrm{Si}$ ) are used as a spacer layer and the substrate, respectively) were taken as $n_{B C B}=1.54, n_{S i}=3.4$, and a simple Drude model was used for gold permittivity (with plasma frequency of $9.02 \mathrm{eV}$ and scattering frequency of $0.038 \mathrm{eV})$ [49]. Figure 3 shows the simulated transmission $\left(T_{x: y}\right.$, where $x=$ test scenario $\mathrm{A}$ or $\mathrm{B} ; y=$ an element in the superpixel) and the polarization extinction ratio $\left(P E R_{x: S}=T_{x: S(0)} / T_{x: S(90)}\right)$ 
for the single-layer Au grating. The transmission dips at the incidence angle $\theta$ of $0^{\circ}$ for test scenario A $\left(\phi=0^{\circ}\right)$ and $\mathrm{B}\left(\phi=90^{\circ}\right)$ are observed at $\sim 3.4 \mu \mathrm{m}$, as shown in Figure 3a,d, which correspond to the first-order surface plasmon resonance (SPR) at the interface between Au grating and Si substrate. As the polar angle of the incident light is increased, this dip at $3.4 \mu \mathrm{m}$ is split into two dips at $\sim 2.9 \mu \mathrm{m}$ and $\sim 3.9 \mu \mathrm{m}$ in case of $\theta=30^{\circ}, \phi=0^{\circ}$, as indicated in Figure 3a, whereas the simulation result for test scenario $\mathrm{B}\left(\theta, \phi=90^{\circ}\right)$ shows no splitting, as shown in Figure $3 \mathrm{~d}$. The momentum matching condition was used to understand the underlying mechanism of the SPR-splitting when a linearly polarized light is incident obliquely.

$$
\begin{gathered}
\vec{k}_{s p}=\vec{k}_{\|} \pm m \vec{G}_{x} \\
\lambda_{s p}=\frac{p}{m}\left(-\sin \theta \cos \phi \pm \sqrt{\sin ^{2} \theta\left(\cos ^{2} \phi-1\right)+n^{2}}\right)
\end{gathered}
$$

where $\vec{k}_{s p}$ and $\lambda_{s p}$ are the SPR wave vector and wavelength, $\vec{k}_{\|}$is the in-plane wave vector of the incident radiation, $\vec{G}_{x}$ is the reciprocal vector of the one-dimensional grating ||$\vec{G}_{x} \mid=2 \pi / p$, where $p$ is the grating period), $m$ is an integer representing the coupling order of SPR, $\theta$ and $\phi$ are the polar and azimuthal angles, and $n$ is the refractive index of the silicon substrate.
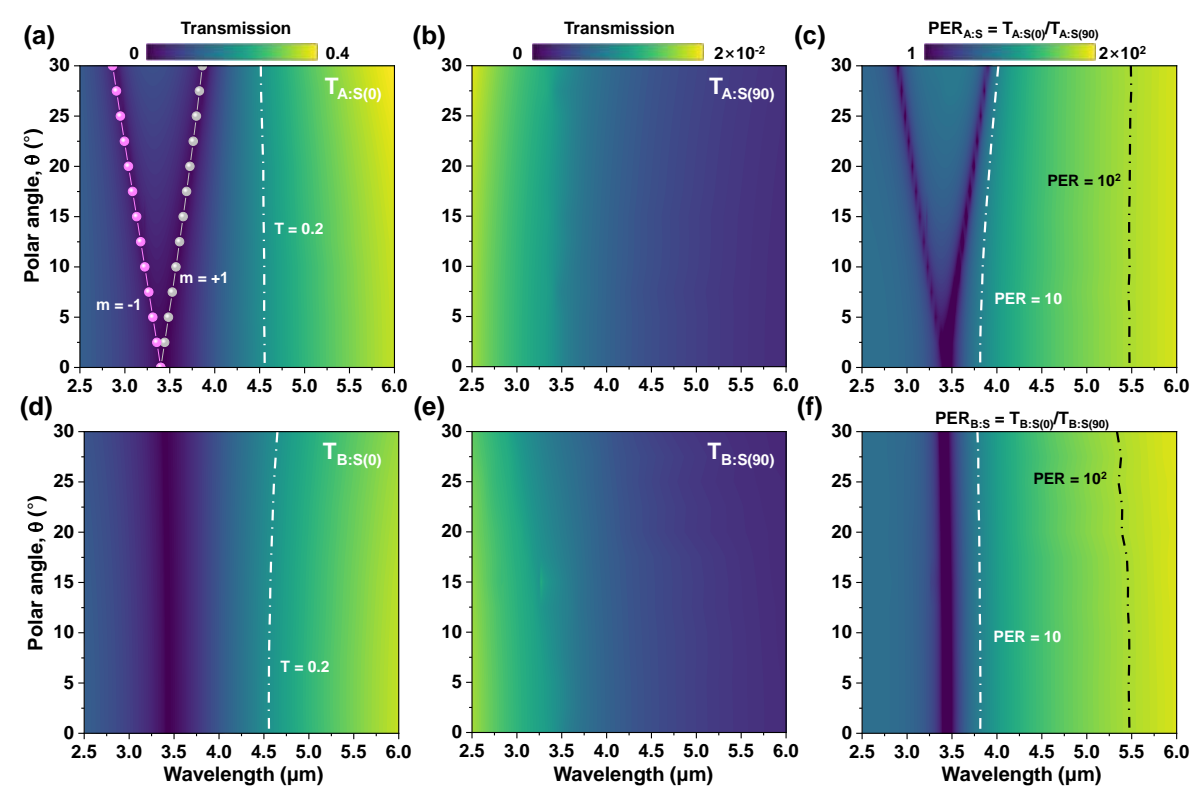

Figure 3. Simulated transmission and polarization extinction ratio of the single-layer Au grating as a function of wavelength $\lambda$ and the polar angle $\theta$ for (a-c) test scenario A (i.e., $T_{A: S(0)}, T_{A: S(90)}, P E R_{A: S}$ ); (d-f) test scenario B (i.e., $\left.T_{B: S(0)}, T_{B: S(90)}, P E R_{B: S}\right)$. The solid lines with symbols represent the wavelengths of the excited surface plasmon resonance (SPR) modes, $\lambda_{s p}(\theta, \phi)$, calculated by Equation (2). Note that the SPR mode with $m=-1$ (red) and +1 (black).

In Figure 3a, the 2-fold degenerate $( \pm 1)$ mode for normal incidence $\left(\theta=0^{\circ}\right)$ is split into two individual SPR modes, $(+1)$ and $(-1)$ at $2 \pi / \vec{k}_{(+1)}$ and $2 \pi / \vec{k}_{(-1)}$, respectively, because $\vec{k}_{(+1)}$ and $\vec{k}_{(-1)}$ have unequal magnitudes when $\theta \neq 0^{\circ}$. However, the 2-fold degenerate $( \pm 1)$ mode for test scenario $B$ $\left(T_{B: S(0)}\right)$ is not split, due to only a very minor increase in the magnitude of $\vec{k}_{( \pm 1)}$ against the polar angle. Note that the electric field perpendicular to the Au gratings lies in the $x y$ plane. Instead, the degenerate $( \pm 1)$ mode is slightly blue-shifted by $\sqrt{n^{2}-\sin ^{2} \theta}$ as the incident light comes in the polar angle $\left(\theta>0^{\circ}\right)$. Figure 3b,e shows the simulated transmission of the single-layer Au gratings oriented at $90^{\circ}$ with polar angles for test scenario $A$ and $B\left(T_{A: S(90)}\right.$ and $\left.T_{B: S(90)}\right) \cdot T_{A: S(90)}$ and $T_{B: S(90)}$ are found to be nearly 
independent of the incidence angle $(\theta)$. Figure $3 c, f$ clearly shows the trend of the calculated PERs, which are closely similar to $T_{A: S(0)}$ and $T_{B: S(0)}$.

Figure 4a shows the simulated transmission of an alternative grating structure where a dielectric spacer layer is inserted between the upper and lower Au grating layers, i.e., the double-layer $\mathrm{Au}$ grating structure $\left(T_{A: D(0)}\right)$. We observed the transmission dip at the same wavelength of single-layer grating $(\sim 3.4 \mu \mathrm{m}$, Figure $3 \mathrm{a})$, which is attributed to the SPR excited at the interface of lower Au grating and $\mathrm{Si}$ substrate in the double-layer structure. As the polar angle increases from 0 to $30^{\circ},(+1) \mathrm{SPR}$ mode red-shifts from $3.4 \mu \mathrm{m}$ to $3.9 \mu \mathrm{m}$, and its transmission intensity changes. On the contrary, an FP cavity mode for normal incidence is created at $\sim 4.5 \mu \mathrm{m}$, which is due to the multiple reflections inside the spacer bounded by two SPR layers. As the polar angle $\theta$ increases from 0 to $20^{\circ}$, the FP resonance peak (cross-symbol) is slightly red-shifted by $2.16 \%$ or less, and the transmission intensity at $\theta=20^{\circ}$ is comparable to $\theta=0^{\circ}$ (Note that the wavelength of FP-peak increases rapidly at larger incidence angles). When the incidence angle is varied $\left(\theta=0^{\circ} \sim 20^{\circ}\right)$, the overall transmission intensity and FP resonance peak for test scenario B is slightly reduced and blue-shifted $(\Delta \lambda<\sim 90 \mathrm{~nm})$, respectively, as shown in Figure $4 \mathrm{~d}$. Figure $4 \mathrm{~b}$,e shows that the simulated transmission of the $D(90)$ sample is almost unaffected by the polar angle and test scenarios, and is about two orders lower than the single-layer structure. Figure $4 \mathrm{c}, \mathrm{f}$ shows a high PER of more than $\sim 10^{3}$ in the region of $4-6 \mu \mathrm{m}$, regardless of polar angles.
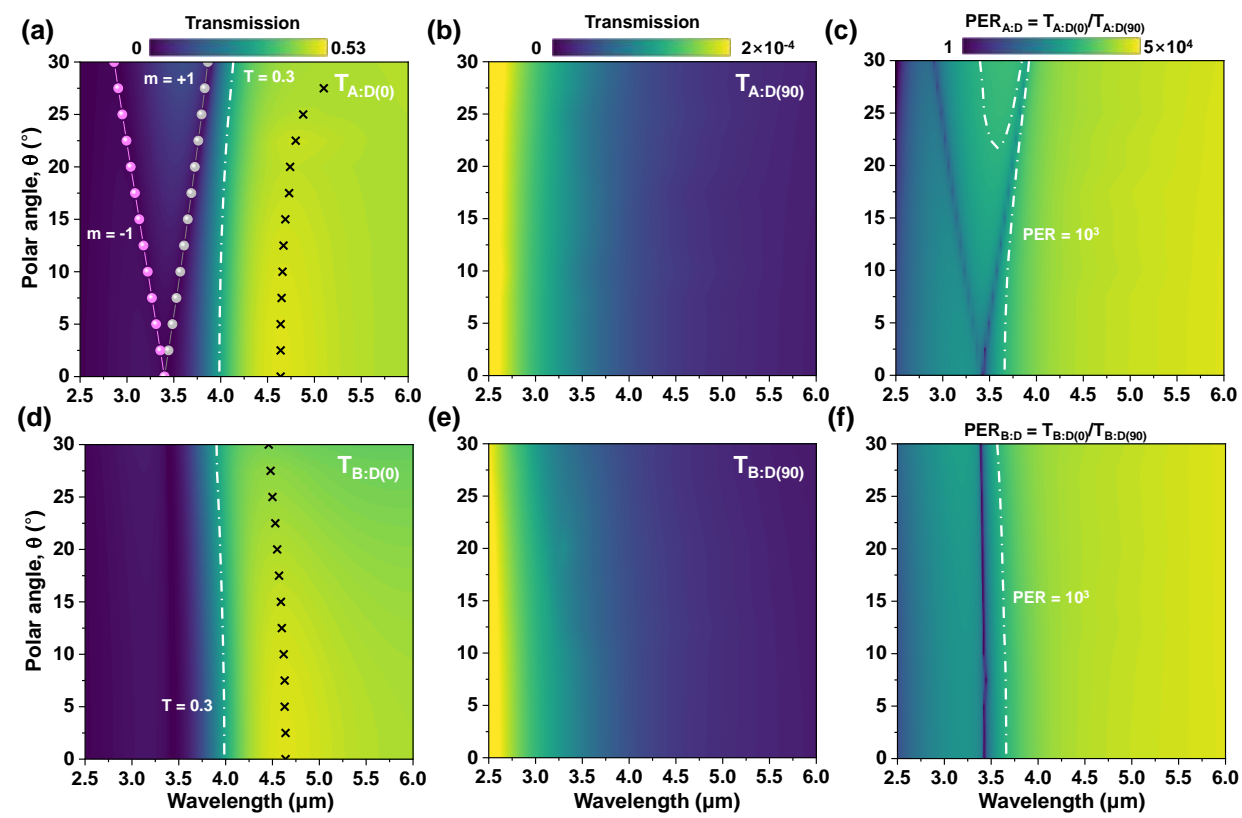

Figure 4. Colormaps of the simulated transmission and polarization extinction ratio of the double-layer Au grating structure for $(\mathbf{a}-\mathbf{c})$ test scenario $\mathrm{A} ;(\mathbf{d}-\mathbf{f})$ test scenario $\mathrm{B}$, which are presented in color as a function of wavelength $\lambda$ and the incidence angle $\theta$.

\section{Polarization Sensitivity Measurement of NIL-Fabricated Double (Single)-Layer Grating}

Polymethyl methacrylate (PMMA) was spun onto a substrate (Si) as a sacrificial layer for liftoff processing, followed by UV nanoimprint resist. The subwavelength gratings were fabricated by imprinting a polydimethylsiloxane (PDMS) mold. After demolding, an anisotropic plasma-reactive ion etch was used to etch the residual UV resist $\left(\mathrm{CHF}_{3} / \mathrm{O}_{2}\right)$ and the PMMA $\left(\mathrm{O}_{2}\right)$. Five nanometer-thick $\mathrm{Cr}$ and $100 \mathrm{~nm}$-thick Au were deposited by e-beam evaporation, followed by liftoff processing with acetone to remove the PMMA layer (Figure 5a). Next, BCB used as the FP optical cavity was spin-coated on the NIL-fabricated single-layer grating sample, followed by curing at $250{ }^{\circ} \mathrm{C}$ for $1 \mathrm{~h}$ (Figure $5 \mathrm{~b}$ ). Finally, the upper Au grating was fabricated by using the same NIL process as described above (Figure 5c). FTIR spectroscopy measurements were carried out to confirm the validity of the simulation 
results, and the polarization extinction ratios (PERs) for test scenario $\mathrm{A}$ and $\mathrm{B}$ were calculated using the measured transmission spectra (i.e., $P E R_{i: j} \equiv T_{i: j(0)} / T_{i: j(90)}$, where $i=$ test scenario A or $\mathrm{B}, j=$ single- or double-layer grating). A commercial wire grid polarizer was used to generate the linearly polarized incident light, and the single- and double-layer grating samples were tilted from 0 to $30^{\circ}$ to mimic the incidence light through the thermal imaging lens.
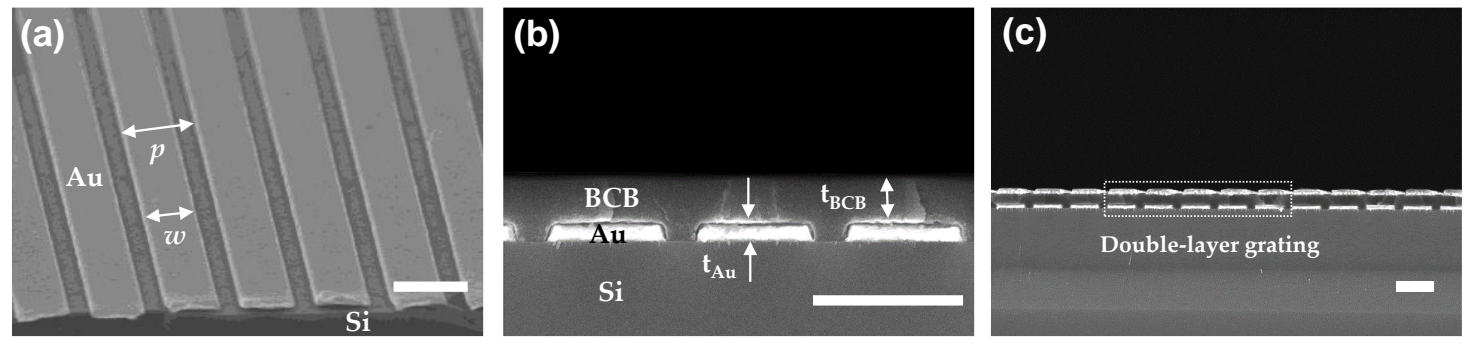

Figure 5. Scanning electron microscope images (tilted- and cross-sectional views) of (a) NIL-fabricated lower Au gratings (single-layer); (b) benzocyclobutane (BCB)-spin coated on the single-layer Au gratings; (c) double-layer Au gratings, followed by NIL-processed upper Au gratings on BCB atop the single-layer grating. The fabricated structural parameters are $p=1.0 \mu \mathrm{m}, w=0.7 \mu \mathrm{m}, t_{\mathrm{Au}}=0.1 \mu \mathrm{m}$, and $t_{\mathrm{BCB}}=0.25 \mu \mathrm{m}$. All scale bars are $1 \mu \mathrm{m}$.

Figure 6 shows the FTIR-measured transmission spectra of the single- and double-layer Au gratings, $S(0)$, and $D(0)$, using various polar angles for test scenario $A$ and $B\left(T_{A: S(0)}, T_{B: S(0)}, T_{A: D(0)}, T_{B: D(0)}\right)$. In test scenario $A$, the 2-fold degenerate SPR dip of $\sim 3.4 \mu \mathrm{m}(m= \pm 1)$ is split into $2.84 \mu \mathrm{m}(m=-1)$ and $3.90 \mu \mathrm{m}(m=+1)$ at the polar angle of $30^{\circ}$ for both single- and double-layer Au gratings, as shown in Figure $6 \mathrm{a}, \mathrm{c}$. This result agrees well with Figure $3 \mathrm{a}$. In test scenario B, the wavelength of SPR mode is also found to be $\sim 3.4 \mu \mathrm{m}$ for normal incidence $\left(\theta=0^{\circ}\right)$ and it is blue-shifted by $\Delta \lambda \approx \sim 40$ nm when the polar angle is increased from 0 to $30^{\circ}$ (Figure $6 \mathrm{~b}$ ). Only the double-layer grating sample appears the transmission peak at $\sim 4.5 \mu \mathrm{m}$ for both scenarios (Figure 6c,d), which originates from FP cavity resonance inside the $\mathrm{BCB}$ spacer $\left(T_{i: D(0)}=0.49\right.$ at $\theta=0^{\circ}, I=\mathrm{A}$ or $\left.\mathrm{B}\right)$, as explained in Section 2. Note that the discrepancy in the peak location of double-layer grating between simulation (Figures 1-5) and experiment (Figure 6), $\Delta \approx 0.2 \mu \mathrm{m}$, is probably due to the imperfections in the fabrication, specifically the misalignment between the upper and lower Au gratings. Detailed information can be found in Appendix A (Figures A7 and A8).

Figure 7 shows the simulated and measured polarization extinction ratios (PERs) of the singleand double-layer Au gratings, which were calculated at a wavelength of $4.5 \mu \mathrm{m}$, corresponding to the FP cavity mode. For test scenario A and B, the simulated PERs of the single- and double-layer Au gratings for the different polar angles were $\sim 40$ and $10^{4}$, respectively, as shown in Figure 7 a. The overall agreement between simulated PER and experimental PER at the FP cavity resonance wavelength is apparent from Figure 6 for the incidence angle range we considered. The measured PERs for the single- and double-layer gratings are found to be $\sim 18$ and $\sim 132$ at normal incidence. By comparison, this corresponds to the polarization sensitivity improvement of the double-layer grating structure by a factor of $\sim 7$ times over the single-layer grating structure, which is enabled by the incorporation of a BCB spacer interposed between two metal grating layers. As a result, TM-transmission is enhanced owing to the FP cavity resonance, and simultaneously, TE-transmission is suppressed due to the double-layer grating configuration, i.e., vertically stacked two identical grating layers. Furthermore, the performance indicator for polarization sensitivity, $P E R_{i: j}(i=$ test scenario $\mathrm{A}$ or $\mathrm{B}, j=$ single- or double-layer grating), in the MWIR regime does not vary with the change of incident angle in some degree. In particular, the relative difference of measured PER for double-layer structure is $\sim 4.7 \%$ $\left(\theta=15^{\circ}\right), \sim 5.8 \%\left(\theta=20^{\circ}\right)$, and $\sim 2.2 \%\left(\theta=25^{\circ}\right)$ as compared to $\theta=0^{\circ}$ (normal incidence). 

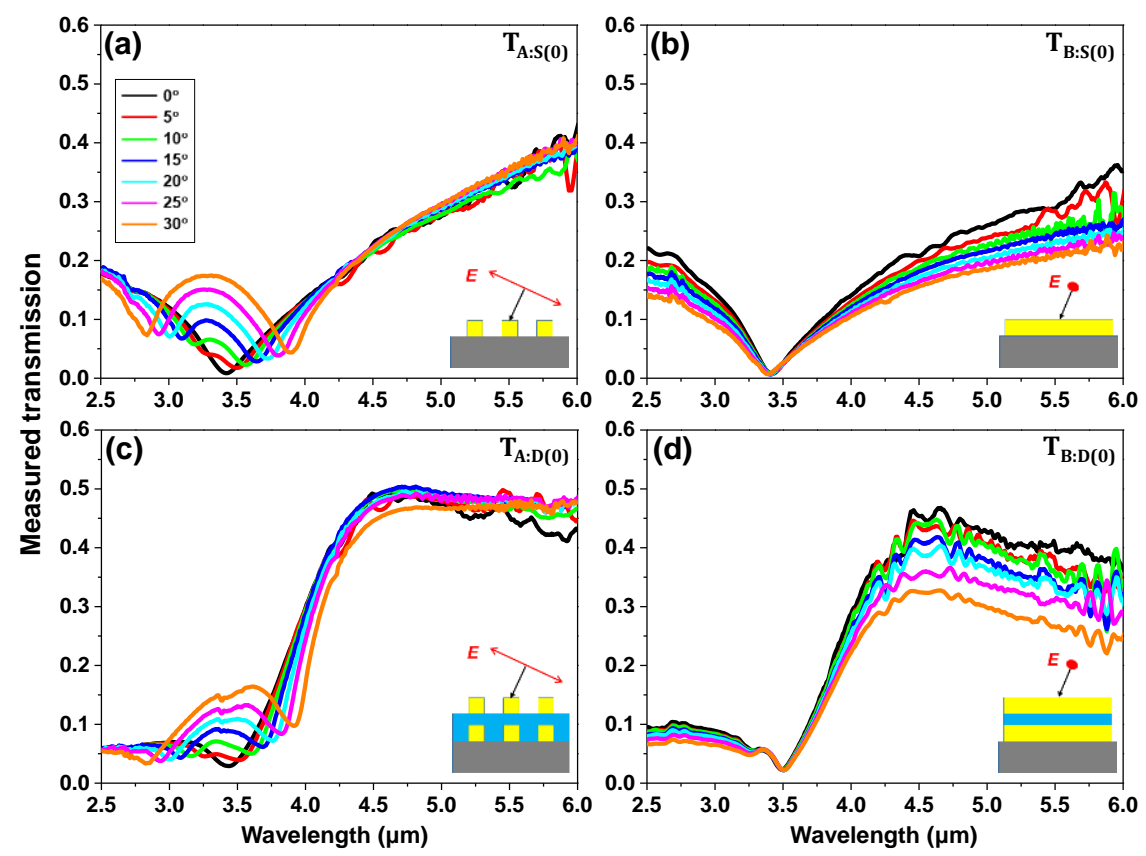

Figure 6. Measured $\theta$-dependent transmission spectra of $0^{\circ}$-orientated $(\mathbf{a}, \mathbf{b})$ single- and $(\mathbf{c}, \mathbf{d})$ double-layer Au gratings for both A, B cases. The fabricated samples were Fourier transform infrared (FTIR)-measured from $\theta=0^{\circ}$ to $\theta=30^{\circ}$ with a step of $5^{\circ}$.
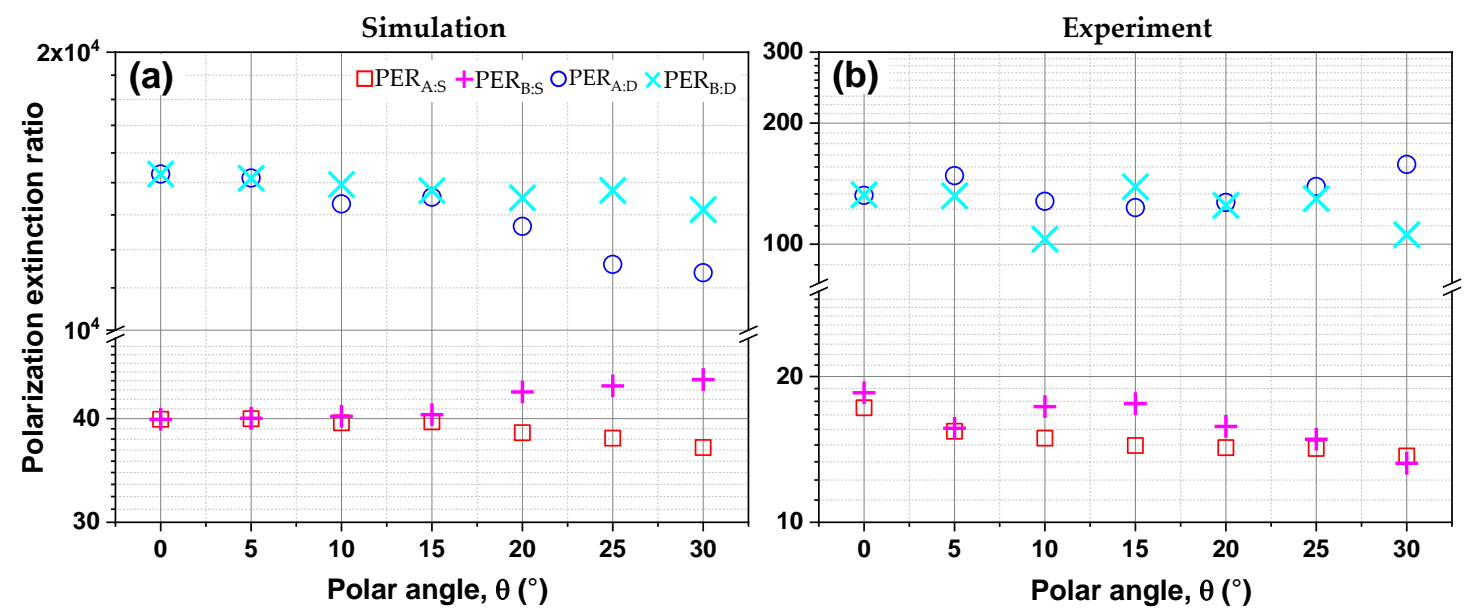

Figure 7. (a) Simulated and (b) measured-polarization extinction ratios (PERs) of the single and double-layer Au gratings as a function of incidence angle $(\theta)$ at $\lambda=4.5 \mu \mathrm{m}$.

\section{Conclusions}

We have experimentally and numerically demonstrated the polarization-sensitivity, and incidence angle-insensitivity of a dielectric spacer bounded by nanoimprint-lithographically fabricated two gold subwavelength grating layers. Using the well-designed spacer serves (1) to create the multiple reflections inside, thereby enhancing the transmittance of IR light polarized perpendicular to the grating direction, (2) to separate the two identical grating layers, thereby suppressing the transmittance of IR light polarized parallel to the grating direction, (i.e., reduced light intensity, due to passing through two identical grating layers), (3) to reduce the cost of a lithography technology resulting from simply decreasing the grating period in a single-layer grating structure (when the grating layers with a large grating period are stacked, the polarization sensitivity can be improved by a factor of $\sim 7$ times over the single-layer grating structures). More interestingly, the polarization extinction ratio (PER, used as the measure of the polarizer performance) of the double-layer grating structure is insensitive 
to the incidence angle to some degree $\left(\left|P E R_{\theta}-P E R_{\theta}=0\right| / P E R_{\theta}=0<6 \%, \theta \leq 25^{\circ}\right)$. We expect that the results drawn from this work could be used as a basis to improve the overall performance of polarization-sensitive elements (constituting the superpixel) and provide a new perspective for the application of IR polarimetric imaging.

Author Contributions: Conceptualization, J.H., Z.K.; formal analysis, J.H., J.J., Z.K.; investigation, J.H., J.J., Z.K.; resources, Y.K., D.-K.K., E.K.K., S.J.L.; writing—original draft preparation, J.H., Z.K.; writing—review and editing, J.H., Z.K., E.K.K., S.J.L.; supervision, Z.K., E.K.K., S.J.L.; funding acquisition, S.J.L. All authors have read and agreed to the published version of the manuscript.

Funding: Korea Research Institute of Standards and Science (KRISS-2020-GP2020-0011); Nano Material Fundamental Technology Development Program (2018069993) through the National Research Foundation of Korea (NRF) funded by the Ministry of Science and ICT; AOARD Grant FA2386-18-1-4021 funded by the US government (AFOSR/AOARD). The AFRL portion of this work was supported by the AOARD Grant FA2386-18-1-4104 funded by the U.S. government (AFOSR/AOARD).

Conflicts of Interest: The authors declare no conflict of interest.

\section{Appendix A}

Figure A1 shows the zeroth and the first diffracted transverse-magnetic (TM)- and transverse-electric (TE)-transmission $\left(T(0)_{T M}, T(0)_{T E}, T( \pm 1)_{T M}, T( \pm 1)_{T E}\right)$, and the polarization extinction ratio (PER) calculated by using $T(0)_{T M}$ and $T(0)_{T E}$ as a function of wavelength and spacer thickness ranging from 0.05 to $2.0 \mu \mathrm{m}$ with a step of $10 \mathrm{~nm}$, which is obtained by COMSOL Multiphysics [50]. A dip, due to the first order Wood-Rayleigh (WR) anomaly (or the first order surface plasmon resonance), is found in the colormaps of $T(0)_{T M}$ and PER(0) regardless of the spacer thickness (Note that $T( \pm 1)=0$ when $\lambda>\lambda_{W R(1)}$, as shown in the lower panel of Figure A1).
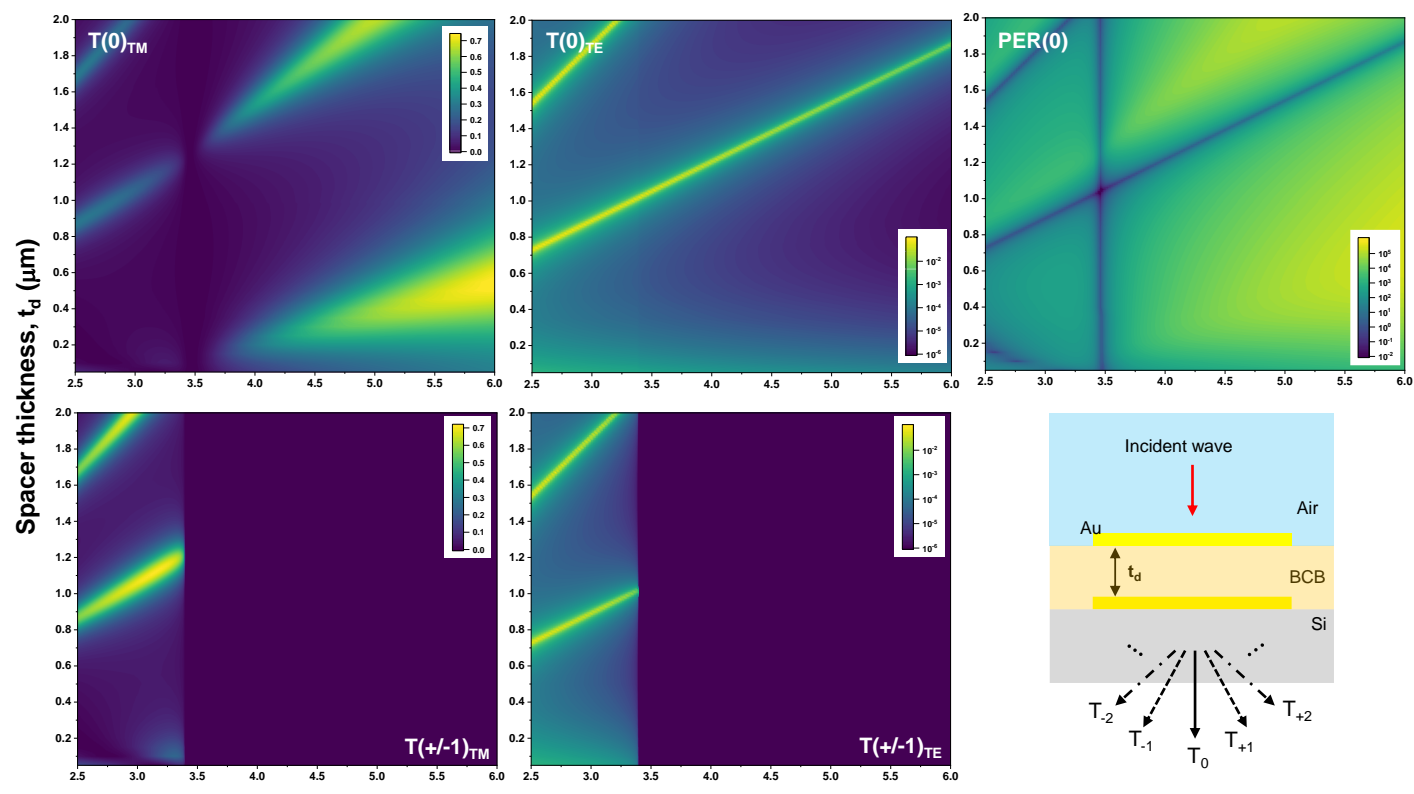

Figure A1. Colormaps of simulated zeroth and first diffracted transverse-magnetic (TM)-, transverse-electric (TE)-transmission, polarization extinction ratio as a function of wavelength $\lambda$ and spacer thickness $t_{\mathrm{d}}$ ranging from $0.05 \mu \mathrm{m}$ to $2 \mu \mathrm{m}$ with a step of $10 \mathrm{~nm}$. Note that the grating period, grating fill factor, Au thickness, and spacer thickness were fixed at $p=1.0 \mu \mathrm{m}, r=0.7$, and $t_{\mathrm{Au}}=0.1 \mu \mathrm{m}$, respectively.

It is commonly known that change in the grating period or grating width affects the polarization sensitivity performance of a single-layer grating (Figures A2-A5). As the grating period of the single-layer grating decreases, TM-transmission $\left(T_{\mathrm{S}(0)}\right)$ is increased, while TE-transmission $\left(T_{\mathrm{S}(90)}\right)$ is suppressed, thereby enhancing the polarization extinction ratio $\left(\mathrm{PER}_{\mathrm{S}}\right)$, as shown in Figure A2 
(upper panel) and Figure A3 (solid line). It can also be observed in the upper panel of Figure A2 that a dip is shifted to a shorter wavelength as the grating period $p$ decreases, which is well known as the feature of the first order Wood-Rayleigh (WR) anomaly, and thus the smaller grating period than $\sim \lambda_{\mathrm{WR}(1)} / n_{\text {sub }}$ is needed to effectively design the single-layer grating structure $\lambda_{\mathrm{WR}(1)}$ is the wavelength of the first order WR and $n_{\text {sub }}$ is the refractive index of the substrate). The complexity, difficulty, and cost are increased to fabricate the grating pattern with such small periodicity, however, the double-layer grating structure has the potential to reduce these drawbacks in the fabrication (lithography) by simply stacking two identical grating layers with a large grating period, as well as to improve the polarization sensitivity, as shown in the lower panel of Figures A2 and A3 (dash-dot line) because a small amount of TE-transmission through the upper grating layer is further reduced by the lower grating layer and simultaneously TM-transmission is improved, due to the FP cavity resonance inside the spacer layer. For this reason, we used a relatively large grating period, $p=1 \mu \mathrm{m}$, to demonstrate high polarization sensitivity and incidence angle-insensitivity of a dielectric spacer bounded by nanoimprint-lithographically fabricated two gold grating layers. Similarly, the grating fill factor (i.e., the ratio of grating width to pitch) was fixed at 0.7 , to have both high PER and practical linear polarizer (i.e., feasible, easy-to-fabricate), as shown in Figures A4 and A5.
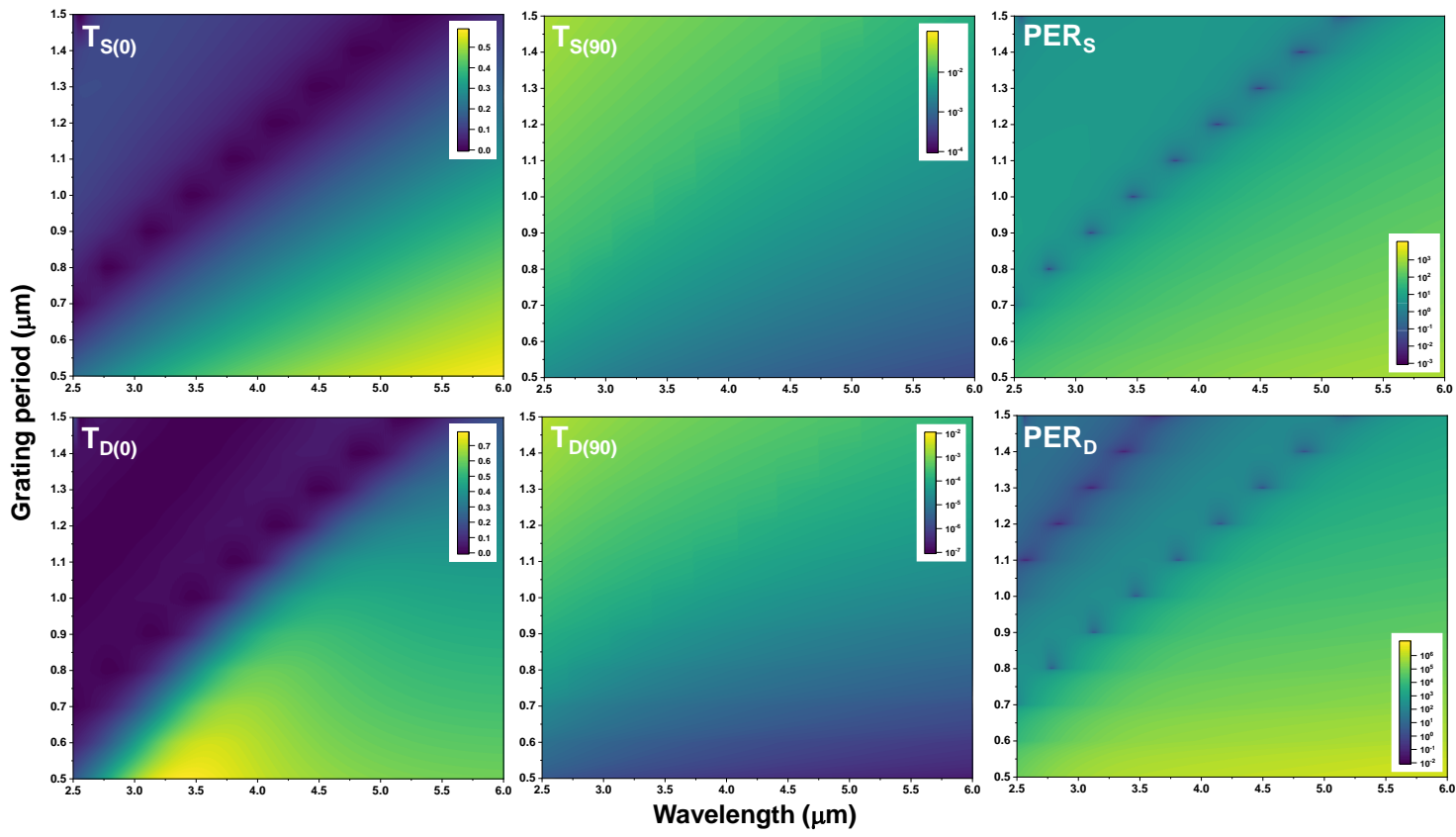

Figure A2. Colormaps of simulated transmission $\left(T_{x(y)}\right.$, where $x=\mathrm{S}$ (single-layer Au grating) or D (double-layer grating), and $y=$ incidence angle) and polarization extinction ratio $\left(P E R_{x}=T_{x(0)} / T_{x(90)}\right.$, where $x=\mathrm{S}$ or D) of the single- and double-layer Au gratings as a function of wavelength $(\lambda)$ and grating period $(p)$. Note that the ratio of grating width to grating period $(r=w / p)$, gold thickness $\left(t_{\mathrm{Au}}\right)$, and spacer thickness $\left(t_{\mathrm{d}}\right)$ were fixed at $r=0.7, t_{\mathrm{Au}}=0.1 \mu \mathrm{m}$, and $t_{\mathrm{d}}=0.25 \mu \mathrm{m}$, respectively.
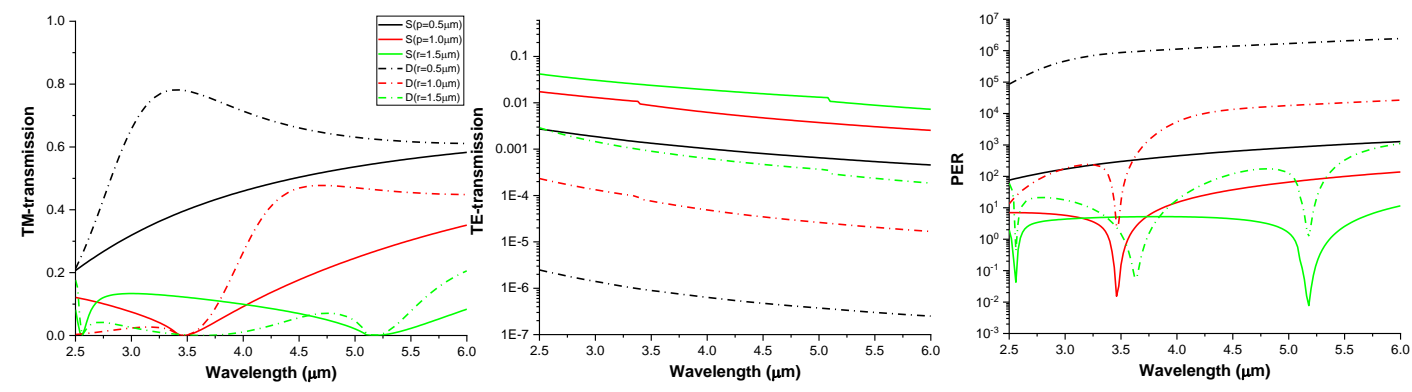

Figure A3. Simulated transmission and polarization extinction ratio of the single- and double-layer Au gratings for the grating period $p=0.5,1.0,1.5 \mu \mathrm{m}$. 

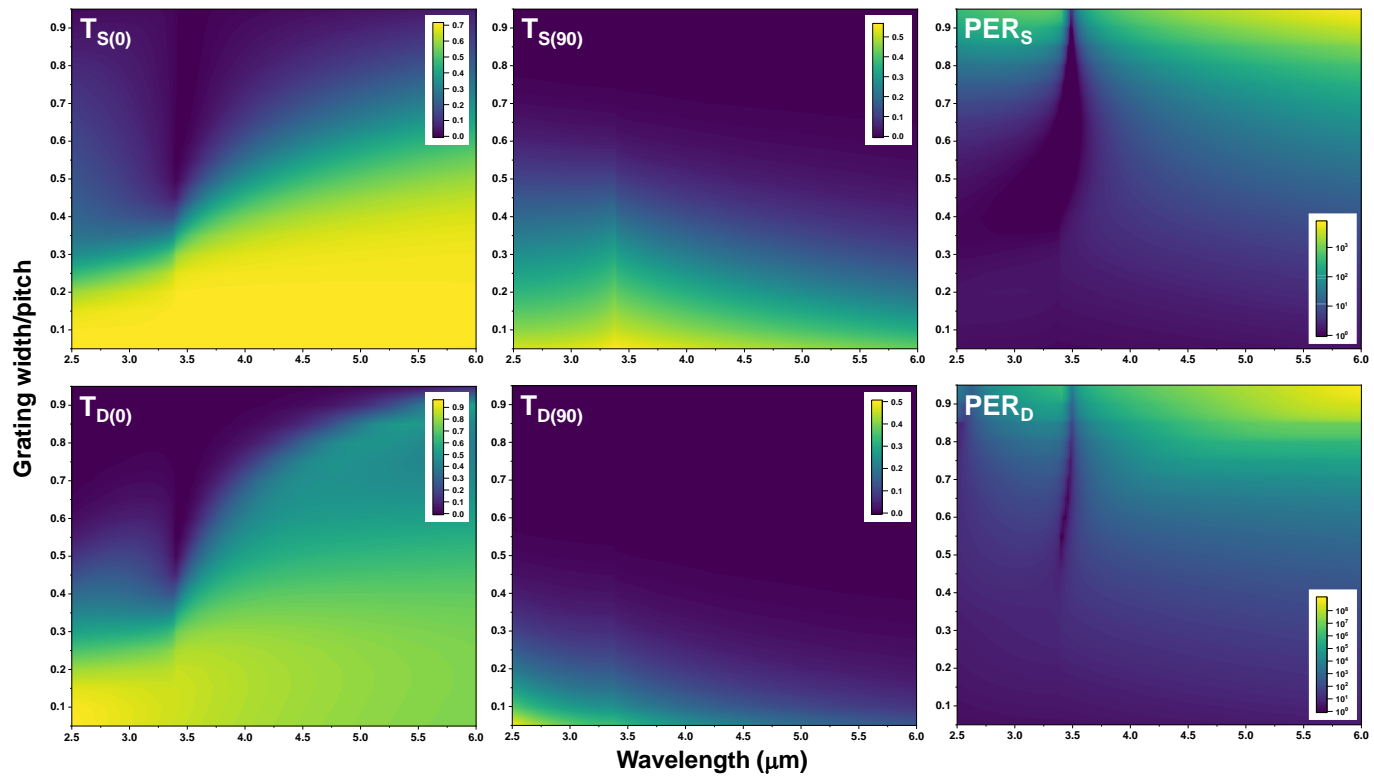

Figure A4. Colormaps of simulated transmission $\left(T_{x(y)}\right.$, where $x=\mathrm{S}$ (single-layer Au grating) or D (double-layer grating), and $y=$ incidence angle) and polarization extinction ratio $\left(P E R_{x}=T_{x(0)} / T_{x(90)}\right.$, where $x=\mathrm{S}$ or $\mathrm{D}$ ) of the single- and double-layer Au gratings as a function of wavelength and ratio of grating width to grating period $(r=w / p)$. Note that the grating period, Au thickness, and spacer thickness were fixed at $p=1.0 \mu \mathrm{m}, t_{\mathrm{Au}}=0.1 \mu \mathrm{m}$, and $t_{\mathrm{d}}=0.25 \mu \mathrm{m}$, respectively.
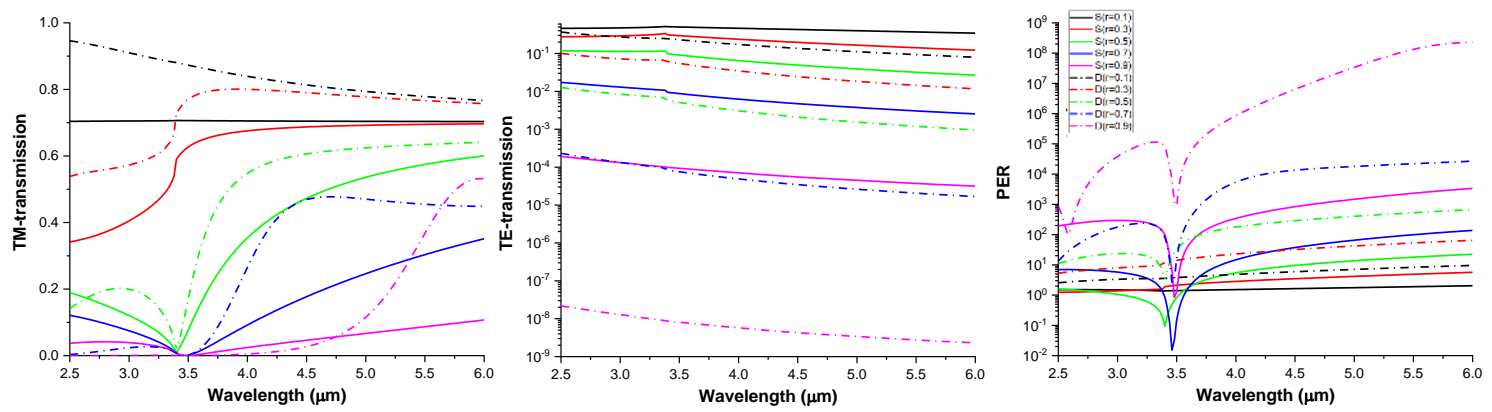

Figure A5. Simulated transmission and polarization extinction ratio of the single- and double-layer Au gratings for $w=0.1,0.3,0.5,0.7,0.9 \mu \mathrm{m}$ ( $r$ in a unit of um can be considered as the width because $p$ is fixed at $1.0 \mu \mathrm{m})$.

Aforementioned, a dip in the transmission spectra for the single- and double-layer grating structures with a pitch of $1 \mu \mathrm{m}$ is observed in the wavelength range of interest (3-5 $\mu \mathrm{m})$, which is attributed to the first order WR anomaly. This dip wavelength is found at $\lambda_{W R(1)}=n_{\text {sub }} \cdot p \approx 3.4 \mu \mathrm{m}$, where $n_{\text {sub }}$ is the refractive index of the substrate. Figure A6 shows the first diffracted transmission ( $T_{\text {tot }}-T_{0}$ ) clearly below the wavelength of $\lambda_{W R(1)}$, and the transmitted light through the single- and double layer gratings is in the zeroth-order diffraction when $\lambda>\lambda_{W R(1)}$. Note that our double-layer grating was designed on the basis of Figures 1 and A1 to have a peak wavelength of $\sim 4.3 \mu \mathrm{m}$.

In order to the influence of alignment-shift of upper Au grating on the polarization sensitivity performance, we performed numerical simulations using COMSOL Multiphysics. We present the TM-, TE-transmission, and polarization extinction ratio using the colormap to show the spectral change clearly. The wavelength, and misalignment between upper and lower Au gratings, are varied continuously. The geometrical parameters of the double-layer grating are as follows: grating period $(p)=1 \mu \mathrm{m}$, grating width $(w)=0.7 \mu \mathrm{m}$, Au thickness $\left(t_{\mathrm{Au}}\right)=0.1 \mu \mathrm{m}$, spacer thickness $\left(t_{\mathrm{d}}\right)=0.25 \mu \mathrm{m}$. The lateral misalignment between two metal gratings is gradually varied from perfectly aligned (Case A: $0 \cdot p=0 \mathrm{~nm}$ ) to completely alignment-shifted upper Au grating (Case D: $p / 2=500 \mathrm{~nm}$ ), as displayed in 
Figure A7. For a minor misalignment, there is a slight difference in the simulation results (Figures A7 and A8) as compared to the perfectly aligned double-layer grating. More interestingly, Figures A7 and $A 8$ show that the overall performance is improved as the lateral misalignment is increased, until the upper Au grating is perfectly misaligned (i.e., Case D). The configuration of Case D is similar to the bilayer grating structure.
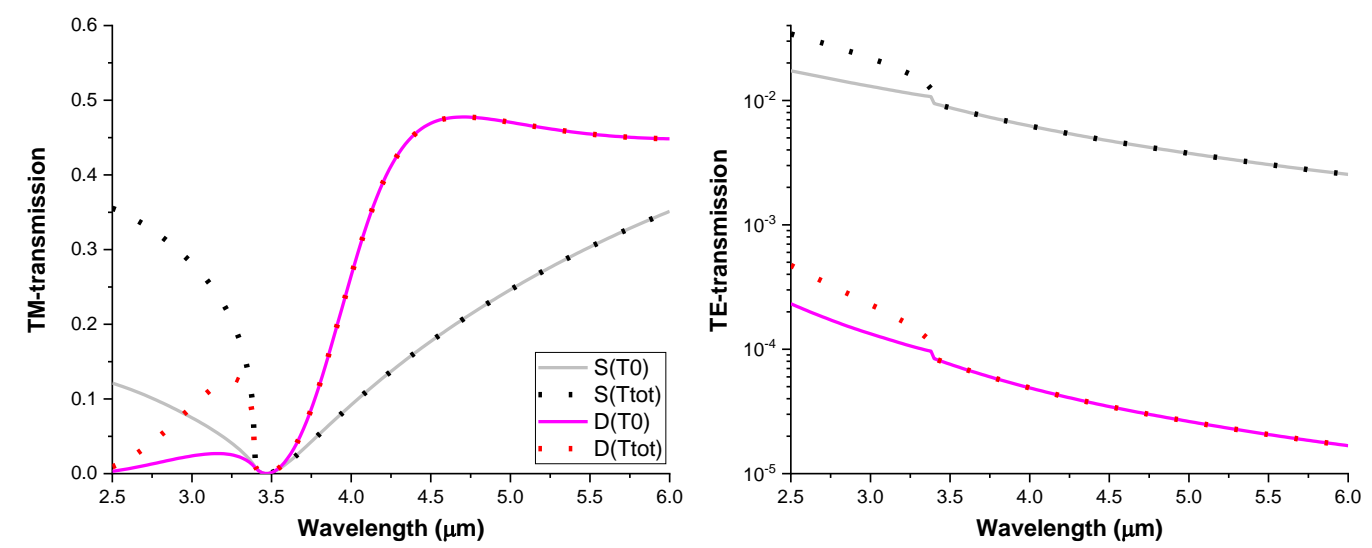

Figure A6. Simulated TM- and TE-transmissions of single-layer grating (S) and double-layer gratings (D) for normal incidence. $T_{0}$ is the zeroth diffracted transmission and $T_{\text {tot }}$ is the total transmission, i.e., the sum of transmission in all diffraction orders. The geometrical parameters of the single- and double-layer gratings are fixed at $p$ (grating period) $=1 \mu \mathrm{m}, w$ (grating width) $=0.7 \mu \mathrm{m}, t_{\mathrm{Au}}(\mathrm{Au}$ thickness) $=0.1 \mu \mathrm{m}$, and $t_{\mathrm{d}}$ (spacer thickness) $=0.25 \mu \mathrm{m}$.

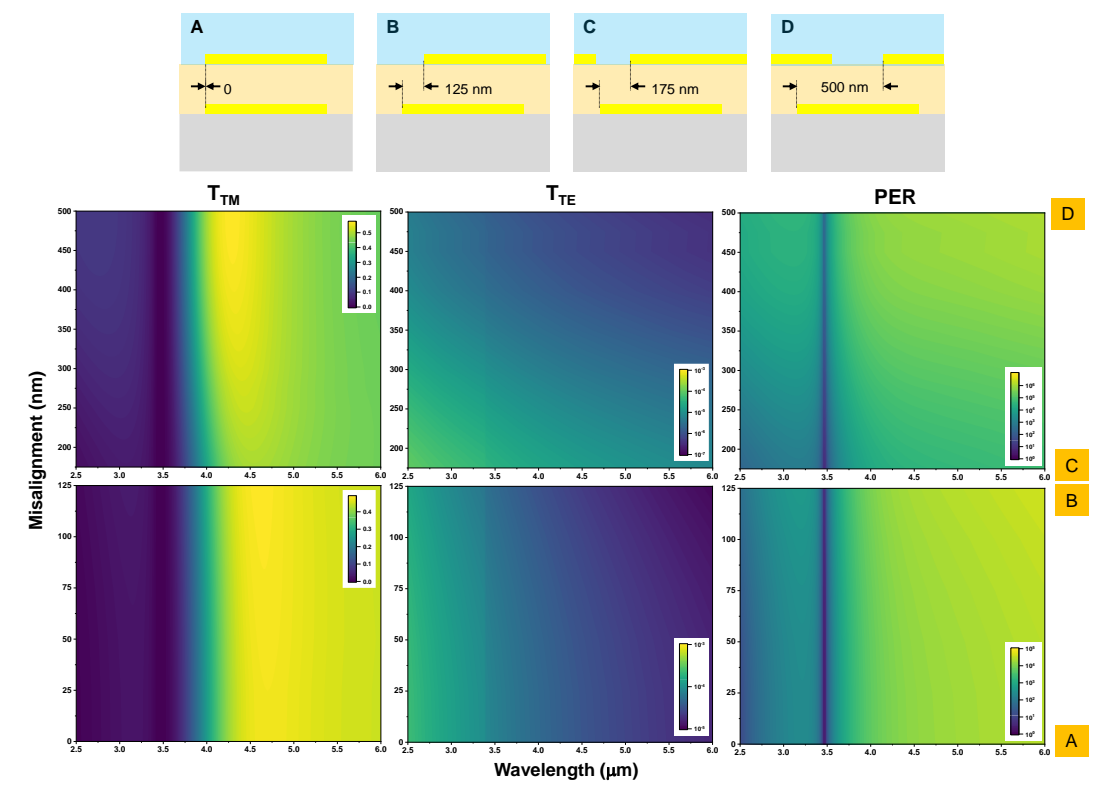

Figure A7. (Upper panel) Schematic view of alignment-shifted top Au grating to bottom Au grating; (Lower panel) colormaps of simulated TM-, TE-transmission, and polarization extinction ratio as a function of wavelength and misalignment between top and bottom Au gratings. The lateral misalignment between two metal gratings is gradually varied from perfectly aligned (i.e., Case A: $0 \mathrm{~nm}$ ) to completely alignment-shifted top Au grating (i.e., Case D: $500 \mathrm{~nm}$ ). The geometrical parameters of the double-layer grating are as follows: grating period $(p)=1 \mu \mathrm{m}$, grating width $(w)=0.7 \mu \mathrm{m}$, Au thickness $\left(t_{\mathrm{Au}}\right)=0.1 \mu \mathrm{m}$, spacer thickness $\left(t_{\mathrm{d}}\right)=0.25 \mu \mathrm{m}$. 

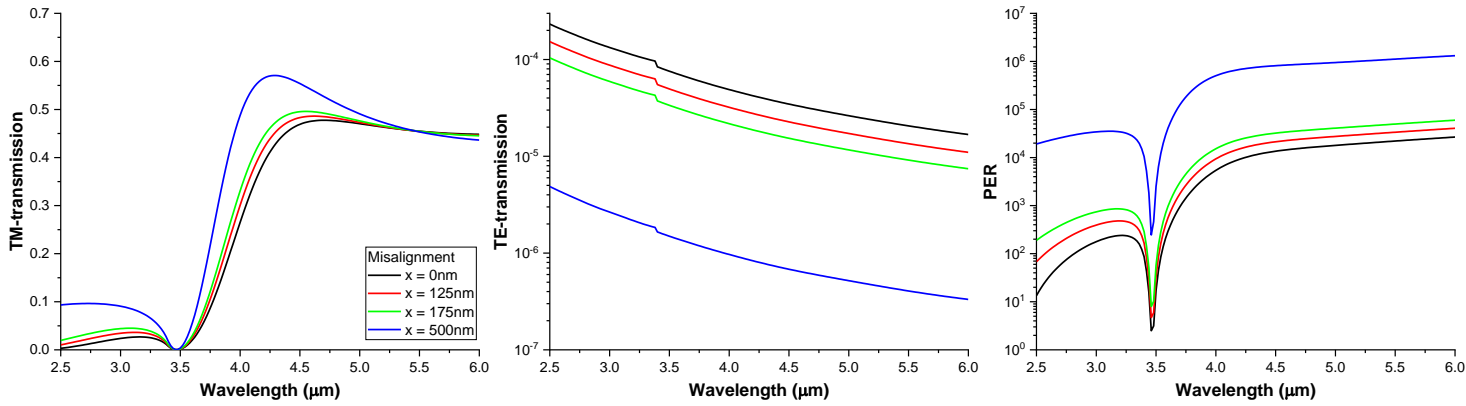

Figure A8. Simulated TM-, TE-transmission, and polarization extinction ratio for alignment-shift of top Au grating ( $0 \mathrm{~nm}, 125 \mathrm{~nm}, 175 \mathrm{~nm}, 500 \mathrm{~nm})$.

\section{References}

1. Tyo, J.S.; Goldstein, D.L.; Chenault, D.B.; Shaw, J.A. Review of passive imaging polarimetry for remote sensing applications. Appl. Opt. 2006, 45, 5453-5469. [CrossRef] [PubMed]

2. Rogne, T.J.; Smith, F.G.; Rice, J.E. Passive target detection using polarized components of infrared signatures. Proc. Soc. Photo Opt. Instrum. Eng. 1990, 1317, 242-251.

3. Shaw, J.A. Degree of linear polarization in spectral radiances from water-viewing infrared radiometers. Appl. Opt. 1999, 38, 3157-3165. [CrossRef] [PubMed]

4. Salomatina-Mottsa, E.; Neela, V.A.; Yaroslavskaya, A.N. Multimodal polarization system for imaging skin cancer. Opt. Spectrosc. 2009, 107, 884-890. [CrossRef]

5. Ogawa, S.; Kimata, M. Wavelength-or polarization-selective thermal infrared detectors for multi-color or polarimetric imaging using plasmonics and metamaterials. Materials 2017, 10, 493. [CrossRef]

6. Felton, M.; Gurton, K.P.; Pezzaniti, J.L.; Chenault, D.B.; Roth, L.E. Measured comparison of the crossover periods for mid-and long-wave IR (MWIR and LWIR) polarimetric and conventional thermal imagery. Opt. Express 2010, 18, 15704-15713. [CrossRef]

7. Gurton, K.P.; Yuffa, A.J.; Videen, G.W. Enhanced facial recognition for thermal imagery using polarimetric imaging. Opt. Lett. 2014, 39, 3857-3859. [CrossRef] [PubMed]

8. Nee, T.W.; Nee, S.M.F. Infrared polarization signatures for targets. Proc. Soc. Photo Opt. Instrum. Eng. 2005, $2469,231-241$.

9. Wilson, J.P.; Schuetz, C.A.; Dillon, T.E.; Eng, D.L.K.; Prather, D.W.; Dillon, T.E. Display of polarization information for passive millimeter-wave imagery millimeter-wave imagery. Opt. Eng. 2012, 51, 091607. [CrossRef]

10. Gurton, K.P.; Dahmani, R. Effect of surface roughness and complex indices of refraction on polarized thermal emission. Appl. Opt. 2005, 44, 5361-5367. [CrossRef] [PubMed]

11. Lewis, G.D.; Jordan, D.L.; Roberts, P.J. Backscattering target detection in a turbid medium by polarization discrimination. Appl. Opt. 1999, 38, 3937-3944. [CrossRef] [PubMed]

12. Tyo, J.S.; Ratliff, B.M.; Boger, J.K.; Black, W.T.; Bowers, D.L.; Fetrow, M.P.; Air, U.S.; Se, A.; Afb, K. The effects of thermal equilibrium and contrast in LWIR polarimetric images. Opt. Express 2007, 15, 15161-15167. [CrossRef] [PubMed]

13. Nordin, G.P.; Meier, J.T.; Deguzman, P.C.; Jones, M.W. Micropolarizer array for infrared imaging polarimetry. J. Opt. Soc. Am. A 1999, 16, 1168-1174. [CrossRef]

14. Andreou, A.G.; Kalayjian, Z.K. Polarization imaging: Principles and integrated polarimeters. IEEE Sens. J. 2002, 2, 566-576. [CrossRef]

15. Gruev, V.; Perkins, R.; York, T. CCD polarization imaging sensor with aluminum nanowire optical filters. Opt. Express 2010, 18, 19087-19094. [CrossRef]

16. Perkins, R.; Gruev, V. Signal-to-noise analysis of Stokes parameters in division of focal plane polarimeters. Opt. Express 2010, 18, 25815-25824. [CrossRef] [PubMed]

17. Antoni, T.; Nedelcu, A.; Marcadet, X.; Facoetti, H.; Berger, V. High contrast polarization sensitive quantum well infrared photodetectors. Appl. Phys. Lett. 2007, 90, 201107. [CrossRef] 
18. Apalkov, V.; Ariyawansa, G.; Perera, A.G.U.; Buchanan, M.; Wasilewski, Z.R.; Liu, H.C. Polarization Sensitivity of Quantum Well Infrared Photodetector Coupled to a Metallic Diffraction Grid. IEEE J. Quantum Electron 2010, 46, 877-883. [CrossRef]

19. Wang, R.; Li, T.; Shao, X.; Li, X.; Gong, H. The simulation of localized surface plasmon and surface plasmon polariton in wire grid polarizer integrated on InP substrate for InGaAs sensor. AIP Adv. 2015, 5, 077128. [CrossRef]

20. Li, Q.; Li, Z.; Li, N.; Chen, X.; Chen, P.; Shen, X.; Lu, W. High-polarization-discriminating infrared detection using a single quantum well sandwiched in plasmonic micro-cavity. Sci. Rep. 2014, 4, 6332. [CrossRef]

21. Young, J.B.; Graham, H.A.; Peterson, E.W. Wire grid infrared polarizer. Appl. Opt. 1965, 4, $1023-1026$. [CrossRef]

22. Porto, J.A.; Garc'1a-Vidal, F.J.; Pendry, J.B. Transmission Resonances on Metallic Gratings with Very Narrow Slits. Phys. Rev. Lett. 1999, 83, 2845-2848. [CrossRef]

23. Lalanne, P.; Hugonin, J.P.; Rodier, J.C. Theory of surface plasmon generation at nanoslit apertures. Phys. Rev. Lett. 2005, 95, 263902. [CrossRef] [PubMed]

24. Pang, Y.; Genet, C.; Ebbesen, T.W. Optical transmission through subwavelength slit apertures in metallic films. Opt. Commun. 2007, 280, 10-15. [CrossRef]

25. Chan, H.B.; Marcet, Z.; Woo, K.; Tanner, D.B.; Carr, D.W.; Bower, J.E.; Pai, C.S. Optical transmission through double-layer metallic subwavelength slit arrays. Opt. Lett. 2006, 31, 516-518. [CrossRef]

26. Tardieu, C.; Estruch, T.; Vincent, G.; Jaeck, J.; Bardou, N.; Collin, S.; Haïdar, R. Extraordinary optical extinctions through dual metallic gratings. Opt. Lett. 2015, 40, 661-664. [CrossRef]

27. Huang, Z.; Parrott, E.P.J.; Park, H.; Chan, H.P.; Pickwell-MacPherson, E. High extinction ratio and low transmission loss thin-film terahertz polarizer with a tunable bilayer metal wire-grid structure. Opt. Lett. 2014, 39, 793-796. [CrossRef]

28. Al, B.; Ekinci, Y.; Solak, H.H.; David, C.; Sigg, H. Bilayer Al wire-grids as broadband and high- performance polarizers. Opt. Express 2006, 14, 2323-2334.

29. York, T.; Gruev, V. Characterization of a visible spectrum division-of-focal-plane polarimeter. Appl. Opt. 2012, 51, 5392-5400. [CrossRef]

30. Ratliff, B.M.; Lacasse, C.F.; Tyo, J.S. Interpolation strategies for reducing IFOV artifacts in microgrid polarimeter imagery. Opt. Express 2009, 17, 9112-9125. [CrossRef]

31. Tyo, J.S.; Lacasse, C.F.; Ratliff, B.M. Total elimination of sampling errors in polarization imagery obtained with integrated microgrid polarimeters. Opt. Lett. 2009, 34, 3187-3189. [CrossRef]

32. Gao, S.; Gruev, V. Image interpolation methods evaluation for division of focal plane polarimeters. Proc. SPIE Int. Soc. Opt. Eng. 2011, 8012, 80120N.

33. Gao, S.; Gruev, V. Bilinear and bicubic interpolation methods for division of focal plane polarimeters. Opt. Express 2011, 19, 26161-26173. [CrossRef]

34. Gao, S.; Gruev, V. Gradient-based interpolation method for division-of-focal-plane polarimeters. Opt. Express 2013, 21, 1137-1151. [CrossRef] [PubMed]

35. LeMaster, D.A.; Hirakawa, K. Improved microgrid arrangement for integrated imaging polarimeters. Opt. Lett. 2014, 39, 1811-1814. [CrossRef] [PubMed]

36. Zhao, X.; Lu, X.; Abubakar, A.; Bermak, A. Novel micro-polarizer array patterns for CMOS polarization image sensors. In Proceedings of the 5th International Conference on Electronic Devices, Systems and Applications, Ras Al Khaimah, UAE, 6-8 December 2016; pp. 1-4.

37. Vaughn, I.J.; Alenin, A.S.; Tyo, J.S. Channeled spatio-temporal stokes polarimeters. Opt. Lett. 2018, 43, 2768-2771. [CrossRef] [PubMed]

38. Zhang, J.; Ye, W.; Ahmed, A.; Qiu, Z.; Cao, Y.; Zhao, X. A novel smoothness-based interpolation algorithm for division of focal plane Polarimeters. In Proceedings of the IEEE International Symposium on Circuits and Systems, Baltimore, MA, USA, 28-31 May 2017; pp. 1-4.

39. Li, N.; Zhao, Y.; Pan, Q.; Kong, S.G. Demosaicking DoFP images using Newton's polynomial interpolation and polarization difference model. Opt. Express 2019, 27, 1376-1391. [CrossRef]

40. Zhang, J.; Luo, H.; Hui, B.; Chang, Z. Image interpolation for division of focal plane polarimeters with intensity correlation. Opt. Express 2016, 24, 20799-20807. [CrossRef]

41. Zhang, J.; Shao, J.; Luo, H.; Zhang, X.; Hui, B.; Chang, Z.; Liang, R. Learning a convolutional demosaicing network for microgrid polarimeter imagery. Opt. Lett. 2018, 43, 4534-4537. [CrossRef] 
42. Park, Y.; Lee, S.; Jeong, B.; Yoon, J. Joint Demosaicing and Denoising Based on a Variational Deep Image Prior Neural Network. Sensors 2020, 20, 2970. [CrossRef]

43. Jiang, T.; Wen, D.; Song, Z.; Zhang, W.; Li, Z.; Wei, X.; Liu, G. Minimized Laplacian residual interpolation for DoFP polarization image demosaicking. Appl. Opt. 2019, 58, 7367-7374. [CrossRef] [PubMed]

44. Zhang, J.; Luo, H.; Liang, R.; Ahmed, A.; Zhang, X.; Hui, B.; Chang, Z. Sparse representation-based demosaicing method for microgrid polarimeter imagery. Opt. Lett. 2018, 43, 3265-3268. [CrossRef] [PubMed]

45. Jeon, J.; Bhattarai, K.; Kim, D.-K.; Kim, J.O.; Urbas, A.; Lee, S.J.; Ku, Z.; Zhou, J. A Low-loss Metasurface Antireflection Coating on Dispersive Surface Plasmon Structure. Sci. Rep. 2016, 6, 36190. [CrossRef] [PubMed]

46. Bhattarai, K.; Silva, S.; Song, K.; Urbas, A.; Lee, S.J.; Ku, Z.; Zhou, J. Metamaterial Perfect Absorber Analyzed by a Meta-cavity Model Consisting of Multilayer Metasurfaces. Sci. Rep. 2017, 7, 10569. [CrossRef]

47. Bhattarai, K.; Ku, Z.; Silva, S.; Jeon, J.; Kim, J.O.; Lee, S.J.; Zhou, J. A Large-Area, Mushroom-Capped Plasmonic Perfect Absorber: Refractive Index Sensing and Fabry-Perot Cavity Mechanism. Adv. Opt. Mater. 2015, 3, 1779-1786. [CrossRef]

48. CST Studio Suite. Available online: https://www.3ds.com/products-services/simulia/products/cst-studiosuite/ (accessed on 19 September 2020).

49. Ordal, M.A.; Long, L.L.; Bell, R.J.; Bell, S.E.; Bell, R.R.; Alexander, R.W.; Ward, C.A. Optical properties of the metals $\mathrm{Al}, \mathrm{Co}, \mathrm{Cu}, \mathrm{Au}, \mathrm{Fe}, \mathrm{Pb}, \mathrm{Ni}, \mathrm{Pd}, \mathrm{Pt}, \mathrm{Ag}$, Ti, and w in the infrared and far infrared. Appl. Opt. 1983, 22, 1099-1119. [CrossRef]

50. COMSOL Multiphysics. Available online: https://www.comsol.com/ (accessed on 19 September 2020).

(C) 2020 by the authors. Licensee MDPI, Basel, Switzerland. This article is an open access article distributed under the terms and conditions of the Creative Commons Attribution (CC BY) license (http://creativecommons.org/licenses/by/4.0/). 